\title{
Boas Práticas em Unidades de Alimentação e Nutrição Escolares de um município do estado do Rio de Janeiro - Brasil
}

\author{
Best Practices in School Food and Nutrition Units \\ of a municipality in the state of Rio de Janeiro - Brazil
}

\author{
Daniele da Silva Bastos Soares ${ }^{1}$ \\ Patrícia Henriques ${ }^{1}$ \\ Daniele Mendonça Ferreira ${ }^{1}$ \\ Patrícia Camacho Dias ${ }^{1}$ \\ Silvia Pereira ${ }^{1}$ \\ Roseane Moreira Sampaio Barbosa ${ }^{1}$
}

${ }^{1}$ Faculdade de Nutrição,

Universidade Federal

Fluminense. R. Mário

Santos Braga 30/4\%,

Valonguinho. 24020

140 Niterói RJ Brasil.

danielebastos@id.uff.br

\begin{abstract}
The scope of this study was to analyze the hygienic-sanitary conditions in nine School Food and Nutrition Units (UANE) of a municipality in the state of Rio de Janeiro (Brazil). A Best Practices in School Food Checklist proposed and validated by Stedefeldt et al., containing items scored and grouped into six thematic blocks (BT) was applied. The total score $(P)$ results per $B T$ and the final score (PF) were obtained using the Best Practices in School Food software ${ }^{\circledR}$. The P results in UANE were compared with the classification of Collegiate Board Resolution No. 275 of 2002 of the National Health Surveillance Agency 2 and PF results were used for UANE classification. All the UANE results were partially suitable for BT building. For BT handlers, processes/procedures, environmental hygiene and equipment for controlled temperature, most had partially adequate and inadequate results. Most UANE were adequate for BT reception. The PF results classified most UANE (66.7\%) as standard health risks, indicating the need for corrective measures for Best Practices in UANE and the supply of safe and healthy meals to schoolchildren.
\end{abstract}

Key words Food safety, Schoolchildren, Checklist, Best practices
Resumo O objetivo deste trabalho foi analisar as condições higiênico-sanitárias em nove Unidades de Alimentação e Nutrição Escolares (UANE) de um município do Rio de Janeiro (Brasil). Uma Lista de Verificação de Boas Práticas na Alimentação Escolar, proposta e validada por Stedefeldt et al., com itens pontuados e agrupados em seis Blocos Temáticos (BT), foi aplicada. Os resultados da pontuação total (P) por BT e da pontuação final (PF) foram obtidos utilizando o Programa Ferramenta para as Boas Práticas na Alimentação Escolar ${ }^{\circ}$. Os resultados da P nas UANE foram comparados com a classificação da Resolução de Diretoria Colegiada no 275, de 2002, da Agência Nacional de Vigilância Sanitária, e os da PF foram utilizados para a classificação das UANE. Todas as UANE se encontraram parcialmente adequadas para o BT edificação. Para os BT manipuladores, processos/procedimentos, higienização ambiental e equipamentos para temperatura controlada, a maioria apresentou resultados parcialmente adequados e inadequados. A maioria da UANE apresentou-se adequada para o BT recebimento. Os resultados da PF classificaram a maior parte das UANE (66,7\%) como risco sanitário regular, indicando a necessidade de realização de medidas corretivas para as Boas Práticas nas UANE e oferta de refeições seguras ao público escolar.

Palavras-chave Segurança alimentar, Escolar, Lista de verificação, Boas práticas 


\section{Introdução}

No Brasil, a alimentação escolar faz parte do Programa Nacional de Alimentação Escolar (PNAE) e é um direito garantido a todos os alunos matriculados na educação básica das redes públicas federal, estadual, distrital e municipal incluindo também aqueles alunos de entidades filantrópicas e conveniadas. Este programa, implantado em 1955, tem como objetivo contribuir para o crescimento e o desenvolvimento biopsicossocial, a aprendizagem, o rendimento escolar e a formação de práticas alimentares saudáveis dos alunos, por meio de ações de Educação Alimentar e Nutricional (EAN) e da oferta de refeições que cubram as suas necessidades nutricionais durante o período letivo ${ }^{1,2}$.

As Unidades de Alimentação e Nutrição (UAN), neste caso, aqui incluídas, as escolares (UANE), devem ter como função primordial a oferta de refeições nutricionalmente adequadas ao perfil da clientela atendida e seguras do ponto de vista higiênico sanitário ${ }^{3}$. Este princípio integra uma das dimensões da Segurança Alimentar e Nutricional (SAN) que considera que o alimento está apto para o consumo, isto é, com segurança, quando não causa doença ou injúria ao consumidor ${ }^{4,5}$.

De um modo geral, as UANE são responsáveis pelo preparo e distribuição de uma grande quantidade de refeições diariamente, sendo de extrema importância, durante estes processos, a adoção de Boas Práticas (BP $)^{6}$. Recentemente, Stedefeldt et al. ${ }^{7}$ desenvolveram e validaram uma Lista de Verificação de Boas Práticas na Alimentação Escolar (LVBPAE) podendo este se constituir em um importante instrumento para o controle de qualidade higiênico-sanitária e SAN das refeições oferecidas aos escolares.

A preocupação com a SAN para coletividade torna-se ainda mais importante quando se refere ao PNAE cuja clientela atendida são alunos de escolas públicas e entidades filantrópicas, sendo estes considerados grupos potencialmente vulneráveis, especialmente sob o ponto de vista socioeconômico.

Diante do exposto, o objetivo deste trabalho foi analisar as condições higiênico-sanitárias de UANE de um município do estado do Rio de Janeiro (RJ) - Brasil.

\section{Materiais e métodos}

Foi realizado um estudo transversal em nove UANE localizadas em escolas de um pólo regional de um município do estado do RJ, no ano de 2014. Estas UANE foram escolhidas em função das escolas serem locais de realização de atividades vinculadas a projetos de pesquisa e extensão da Faculdade de Nutrição da Universidade Federal Fluminense (UFF).

Para avaliação das condições higiênico-sanitárias das UANE, foi aplicada uma LVBPAE proposta e validada por Stedefeldt et al. ${ }^{7}$ e específica para o segmento da alimentação escolar, com atendimento das exigências da Resolução no 38, de 2009, do Fundo Nacional de Desenvolvimento da Educação ${ }^{8}$ e da Resolução de Diretoria Colegiada (RDC) no 216, de 2004, da Agência Nacional de Vigilância Sanitária (Anvisa) ${ }^{6}$.

O preenchimento da LVBPAE foi realizado por estudantes, previamente habilitados, do curso de graduação em nutrição da UFF. A partir da observação visual das UANE, as seguintes opções de resposta em cada item da lista foram marcadas: "sim", caso a UANE atendesse à questão; "não", no caso de não atendimento ao item; e "não se aplica", caso o item não fosse aplicado à UANE.

A LVBPAE é composta por 99 itens pontuados e agrupados em seis Blocos Temáticos (BT). A cada um dos itens, que recebeu como opção "sim", foram atribuídas notas que variaram de um a oito, conforme o grau de risco e importância para a SAN. Para os itens que representam condições ou situações que evitam a multiplicação de microrganismos, foram atribuídas a nota oito. Para os que evitam a sobrevivência de microrganismos, foram atribuídas a nota quatro. Nas ocasiões que evitam a contaminação cruzada por contato direto com o alimento, foram atribuídas a nota dois e, para os que evitam a contaminação cruzada, sem contato direto com o alimento, foram atribuídas a nota um. As respostas assinaladas na LVBPAE como alternativa não, que caracterizaram a não conformidade do item às $\mathrm{BP}$, receberam a nota zero ${ }^{7}$.

Os BT da LVBPAE receberam uma pontuação de peso (k) de acordo com o grau de risco de contaminação das situações ou condições pertencentes a esse bloco. O peso consiste em um valor constante, cuja somatória resulta em 100 e que atua como um multiplicador nos blocos, dando maior pontuação aos considerados de maior risco. Os BT estabelecidos e seus respectivos pesos considerados foram: Edificações, $\mathrm{k}=$ 
10; Equipamentos para Temperatura Controlada, $\mathrm{k}=15$; Manipuladores, $\mathrm{k}=25$; recebimento, $\mathrm{k}=$ 10; Processos e Procedimentos, $\mathrm{k}=30$ e Higienização Ambiental, $\mathrm{k}=10^{7}$.

Para determinação da pontuação total (P) por BT, em cada UANE, foi utilizada a seguinte equação ${ }^{7}$ :

$\mathrm{P}=\mathrm{TS} /\left(\sum \mathrm{TP}-\sum \mathrm{NA}\right) \mathrm{x} \mathrm{K}$

Onde TS é o total de pontos obtidos; $\sum \mathrm{TP}$, o total de pontos possíveis; $\sum \mathrm{NA}$, o total de pontos atribuídos a itens de verificação "não aplicáveis"; e K é o peso atribuído ao bloco temático $\left(\sum \mathrm{k}=\right.$ 100).

Os resultados foram expressos em percentuais de adequação das $\mathrm{BP}^{7}$.

A pontuação final (PF), das UANE avaliadas, foi obtida a partir do somatório da pontuação dos seis blocos temáticos $(\mathrm{PF}=\mathrm{P} 1+\mathrm{P} 2+\mathrm{P} 3+$ $\mathrm{P} 4+\mathrm{P} 5+\mathrm{P} 6)^{7}$.

Os resultados da pontuação total $(\mathrm{P})$ por BT e da pontuação final (PF) nas UANE foram obtidos utilizando o Programa Ferramenta para as Boas Práticas na Alimentação Escolar ${ }^{\varpi}$.

Os resultados da pontuação total $(\mathrm{P})$ por cada BT nas UANE foram comparados com os critérios de classificação da RDC no 275 de 2002 da Anvisa ${ }^{9}$, que estabelece como grupo A (adequado - > 75\% de conformidade dos itens), grupo B (parcialmente adequado - 51-75\% de conformi-

Tabela 1. Classificação de risco sanitário atribuída às UANE após pontuação final (PF) obtida por meio da LVBPAE.

\begin{tabular}{lr}
\hline \multicolumn{1}{c}{ Classificação } & \multicolumn{1}{c}{ PF (\%) } \\
\hline Situação de risco sanitário muito alto & 0 a 25 \\
Situação de risco sanitário alto & 26 a 50 \\
Situação de risco sanitário regular & 51 a 75 \\
Situação de risco sanitário baixo & 76 a 90 \\
Situação de risco sanitário muito baixo & 91 a 100 \\
\hline Fonte: Stedefeldt et al. ${ }^{7}$.
\end{tabular}

dade dos itens) e grupo C inadequado $<50 \%$ de conformidade dos itens). Para classificação final das UANE, conforme o risco sanitário, foram utilizados os critérios apresentados na Tabela 1.

Este estudo foi conduzido em conformidade com as normas estabelecidas pelo Comitê de Ética em Pesquisa (CEP) da Universidade Federal Fluminense e Comitê de Ética em Pesquisa da Faculdade de Medicina/ Hospital Antônio Pedro.

\section{Resultados}

De acordo com os resultados da Tabela 2, verifica-se que todas as UANE se encontraram no grupo B (parcialmente adequado) para o BT edificação. Para os BT manipuladores, processos/procedimentos e higienização ambiental, a maioria $(n=8)$ apresentou resultados enquadrados nos grupos B (parcialmente adequado) e C (inadequado). Mais da metade delas $(\mathrm{n}=5)$ também apresentou para o BT equipamentos para temperatura controlada resultados nos grupos B (parcialmente adequado) e C (inadequado). Por outro lado, a maior parte da UANE $(\mathrm{n}=8)$ encontrou-se no grupo A (adequado) para o BT recebimento.

Na Tabela 3 observa-se que a maioria $(n=6)$ das UANE analisadas apresentou situação de risco sanitário regular, um pequeno percentual $(\mathrm{n}=$ 2) foi classificado com situação de risco sanitário baixo e apenas uma UANE apresentou risco sanitário muito alto.

\section{Discussão}

A oferta de alimentos e preparações de qualidade higiênico-sanitária integra a dimensão alimentar da SAN que está relacionada à garantia da qualidade biológica, sanitária, nutricional e tecnológi-

Tabela 2. Pontuação total (P) por Bloco Temático (BT) das Unidades de Alimentação e Nutrição Escolares (UANE) de um município do estado do Rio de Janeiro, após aplicação da LVBPAE.

\begin{tabular}{|c|c|c|c|c|c|c|c|c|c|}
\hline \multirow{2}{*}{ BT } & \multicolumn{9}{|c|}{$\mathrm{P}(\%)$ das UANE } \\
\hline & 1 & 2 & 3 & 4 & 5 & 6 & 7 & 8 & 9 \\
\hline Edificações e Instalações & 58,1 & 73,7 & 63,1 & 70,9 & 72,1 & 52,6 & 63,6 & 71,3 & 67,1 \\
\hline Equipamentos para temperatura controlada & 84,6 & 100,0 & 100,0 & 54,5 & 23,5 & 46,7 & 55,6 & 63,6 & 84,6 \\
\hline Manipuladores & 50,0 & 66,7 & 70,0 & 75,0 & 50,0 & 50,0 & 66,7 & 53,8 & 100,0 \\
\hline Recebimento & 100,0 & 100,0 & 90,9 & 100,0 & 100,0 & 0 & 100,0 & 100,0 & 100,0 \\
\hline Processos/Procedimentos & 44,7 & 62,0 & 55,8 & 54,2 & 42,6 & 76,8 & 53,6 & 50,3 & 67,1 \\
\hline Higienização ambiental & 55,0 & 79,5 & 36,8 & 40,5 & 29,7 & 34,2 & 44,1 & 54,8 & 28,6 \\
\hline
\end{tabular}


Tabela 3. Classificação de risco sanitário (PF) das UANE de um município do estado do RJ, após aplicação de LVBPAE.

\begin{tabular}{lrr}
\hline \multicolumn{1}{c}{ PF (\%) } & n & \% \\
\hline Situação de risco sanitário muito alto (0 a 25) & 1 & 11,1 \\
Situação de risco sanitário alto (26 a 50) & 0 & 0,0 \\
Situação de risco sanitário regular (51 a 75) & 6 & 66,7 \\
Situação de risco sanitário baixo (76 a 90) & 2 & 22,2 \\
Situação de risco sanitário muito baixo (91 a 100) & 0 & 0,0 \\
\hline $\mathrm{n}=9$ &
\end{tabular}

ca dos alimentos, bem como seu aproveitamento, estimulando práticas alimentares e estilos de vida saudáveis que respeitem a diversidade étnica e racial e cultural da população brasileira ${ }^{4}$.

A análise do risco de contaminação da alimentação escolar permite detectar com maior exatidão onde é necessário agir, identificando qual etapa do processo produtivo interfere na segurança do alimento. A maior parte das UANE apresentou resultados inadequados e parcialmente inadequados para quase todos os BT avaliados, com exceção do recebimento.

Para o BT edificações e instalações, os itens que contribuíram para as inadequações foram a localização da UANE, local para higienização das mãos, forros e tetos, portas, janelas, lâmpadas e ventilação. Resultados similares foram encontrados em outros estudos tais como tetos e forros descascados com presença de mofo e umidade, janelas sem telas, janelas e portas em má conservação, ausência de proteção nas luminárias contra queda e explosão das lâmpadas e iluminação e ventilação ineficientes nas áreas de preparo $^{10-15}$.

As principais inadequações encontradas no BT equipamentos para temperatura controlada foram a ausência de equipamentos com visor de temperatura, termômetros para aferição da cadeia fria e quente e do balcão térmico de distribuição das refeições. Estes resultados corroboram com os achados de diversos estudos que também avaliaram as BP em UANE ${ }^{10-15}$.

Com relação ao BT manipuladores, os itens que mais influenciaram os resultados foram àqueles relacionados a não utilização ou utilização incompleta de uniforme, à ausência de proteção para os cabelos, presença de calçados impróprios, o uso de adornos e à falta de realização de exames de saúde periódicos. Outros estudos também encontraram inadequações quanto à avaliação dos manipuladores de escolas e creches, destacando-se que os itens mais frequentes foram o uso incorreto do uniforme, ausência da touca de proteção para cabelos e de realização de exames médicos periódicos ${ }^{10-12,14}$.

$\mathrm{Na}$ avaliação do BT recebimento, destacase como limitação deste estudo a ausência de acompanhamento das atividades relacionadas a este item uma vez que, durante a aplicação da LVBPAE, não houve entrega de gêneros alimentícios. O preenchimento deste BT foi realizado a partir da indagação às merendeiras se os resultados encontrados podiam não corresponder à rotina destas atividades, uma vez que a maioria das merendeiras podia ter relatado os procedimentos adequados durante o recebimento dos gêneros. Apenas uma UANE apresentou resultado inadequado para este BT devido às respostas relacionadas a procedimentos não conformes. Alguns resultados da literatura vão de encontro ao presente estudo destacando a ausência de verificação de temperatura, validade e integridade das embalagens dos gêneros alimentícios durante o recebimento dos mesmos ${ }^{11,13}$.

Os aspectos que mais contribuíram negativamente para o BT processos/procedimentos relacionaram-se à higienização inadequada das mãos dos manipuladores, presença de caixas de papelão ou madeira no estoque seco, armazenamento inadequado dos alimentos e preparações nos refrigeradores, falta de controle e registro de temperaturas da cadeia fria e quente e do armazenamento das amostras das preparações. Estudos desenvolvidos em UANE também verificaram que, em relação aos processos e procedimentos, as inconformidades mais frequentes incluíam o processo de higienização das mãos dos manipuladores e a inexistência de um sistema de controle das temperaturas das refeições servidas ${ }^{11-14}$.

Outro ponto importante destacado por Rebelo ${ }^{14}$ foi a ausência de lavatórios específicos para a higienização das mãos nas UANE avaliadas. Este item, relacionado à estrutura física inadequada da UANE, traz como consequência a inadequação na higienização das mãos dos manipulado- 
res durante o preparo das refeições, avaliados no item BT manipuladores ${ }^{13}$. Além disso, problemas relacionados às compras da administração pública também podem ser um complicador para a disponibilidade permanente de produtos de limpeza e desinfecção nas UANE.

Com relação ao BT higienização ambiental, foram verificados procedimentos inadequados na lavagem, higienização e armazenamento dos utensílios, equipamentos, panos e esponjas, além da presença de pragas. Outros estudos também verificaram que os utensílios não foram submetidos aos processos adequados de lavagem e higienização e, além disso, eram armazenados em locais inapropriados ${ }^{11-14}$.

De um modo geral, sabe-se que os BT manipuladores, processos/procedimentos e higienização ambiental estão diretamente ligados à figura do manipulador de alimentos. Nesse sentido, a qualidade dos produtos e serviços de alimentação pode ser interpretada como o resultado de um conjunto de condições e cuidados, compreendidos em toda a cadeia produtiva, desde a obtenção da matéria-prima até o momento da sua utilização. Dentre um dos componentes que podem afetar essa qualidade, destaca-se o papel da merendeira durante o processo produtivo de refeições. Desta forma, considerando a relevância desta profissional para a promoção das BP em UANE, torna-se essencial a realização de oficinas de formação ${ }^{16}$ conforme previsto na legislação sanitária brasileira ${ }^{6}$.

No entanto, essas oficinas têm sido consideradas ineficientes quando baseadas apenas na transmissão do conhecimento conferindo às merendeiras o mero papel de observadores e absorvedores, o que dificulta a mudança de hábito na atividade laboral. Para favorecer a mudança de atitude é recomendável que as metodologias de ensino-aprendizagem sejam problematizadoras, críticas, significativas e produtoras de conhecimento. Para tanto, os temas escolhidos deverão decorrer do cotidiano e experiências vividas pelas merendeiras em seus locais de trabalho, para que possam responder as questões inerentes ao seu universo. Portanto, a educação permanente, pautada na discussão da realidade, e valorização do profissional poderá desencadear a ressignificação das práticas com maior probabilidade de mudanças no exercício profissional ${ }^{17}$.

Um trabalho realizado com merendeiras na Bahia (Brasil) exemplifica esta mudança de paradigma, onde inicialmente realizou-se uma "sondagem" dos temas que mais interessavam aos participantes e, posteriormente, tomaram como referencial teórico a pedagogia sociocrítica, que tem por objetivo "discordância construtiva para implementar as habilidades de decisão e realização”. Os recursos metodológicos utilizados foram o teatro, exposição dialogada, oficinas, gincana, experiências práticas, jogos interativos e atividades do grupo. A avaliação do curso pelas merendeiras apontou "satisfação, boa compreensão do conteúdo e adequação entre a metodologia e o referencial teórico"16.

Com relação à classificação de risco sanitário das UANE avaliadas, observou-se que 22\% delas foi classificada com situação de risco sanitário baixo, a maioria (67\%) apresentou situação de risco sanitário regular e $11 \%$ apresentou risco sanitário muito alto. Estudo realizado em UANE da Paraíba encontrou 4,4\% classificadas em risco sanitário baixo, $48,3 \%$ em risco sanitário regular, $24,3 \%$ em risco sanitário alto e $24,0 \%$ em risco sanitário muito alto ${ }^{18}$.

Como esta LVBPAE foi baseada na RDC 216 da Anvisa $^{6}$, que estabelece as condições higiênico-sanitárias para todos os serviços que oferecem alimentos ao público (lanchonetes, restaurantes, cozinhas industriais, buffets, padarias, pastelarias, confeitarias e outros), diversos itens foram pontuados como inadequados, como a presença de lavatórios exclusivos para a lavagem das mãos das merendeiras; sistemas de proteção de luminárias; controle de temperatura da cadeia fria e quente do processo produtivo de refeições; coleta e armazenamento de amostras de preparações alimentares; presença de manual de Boas Práticas e Procedimentos Operacionais Padronizados (POP); etiquetagem dos produtos pós manipulação e descarte de pano de limpeza a cada duas horas.

A presença destes itens na lista se distancia da realidade das UANE que, de um modo geral, possuem estrutura física de uma cozinha doméstica e um quantitativo de merendeiras muitas vezes inadequado, além da ausência diária do profissional nutricionista para um controle higiênicosanitário mais efetivo do processo produtivo de refeições. Esta observação pode ser considerada um fator limitante deste estudo uma vez que diversos itens da LVBPAE, pontuados em inadequados como resposta, podem ter contribuído para uma pior classificação das UANE quanto ao risco sanitário.

Com base nos itens acima descritos, algumas perguntas podem ser feitas: quem ficará responsável pelo controle de temperatura da cadeia fria e quente? Como manter a temperatura das preparações já que não estão disponíveis balcões tér- 
micos, pass through e geladeiras em quantidades adequadas? As UANE têm freezer em quantidade suficiente para o armazenamento das amostras por $72 \mathrm{~h}$ ? O orçamento prevê a compra de panos de limpeza que devem ser descartados a cada 2 horas? Além disso, não há um responsável técnico diariamente na UANE para supervisão das atividades e elaboração de manual de BP e dos POP.

As questões apresentadas não invalidam os resultados apresentados, porém é importante utilizar essa lista com precaução para que os resultados não sejam enviesados. De um modo geral, uma LVBPAE deve ser um instrumento objetivo e de fácil aplicação para um diagnóstico rápido e eficaz da UANE de tal modo que as medidas corretivas possam ser planejadas e executadas na sequência da aplicação.

Recentemente, a Anvisa instituiu a Resolução RDC n ${ }^{\circ} 49$ de $2013^{19}$ visando estabelecer normas de interesse sanitário para a regularização do exercício de atividade dos microempreendedores individuais (MEI). Esta resolução define que é de competência das vigilâncias sanitárias (VISA) municipais a classificação do risco sanitário das atividades desenvolvidas pelos MEI, bem como o processo de licenciamento sanitário dessas empresas. Nesse contexto, faz-se necessário construir um instrumento norteador para padronização das ações da VISA onde o roteiro de inspeção leve em consideração as características de uma UANE, que tem estrutura física e quantitativa de pessoal diferente das institucionais.

\section{Conclusão}

Verificou-se que a maioria das UANE foi classificada com risco sanitário regular. Sendo assim, algumas medidas corretivas tornam-se importantes para a melhoria das condições higiênicosanitárias nas UANE avaliadas tais como, reformas estruturais, instalação de telas milimétricas nas janelas, instalação de luminárias blindadas, aquisição de termômetros e de balcões térmicos, diminuição no tempo de espera da distribuição das refeições expostas à temperatura ambiente e aumento da frequência e adaptação metodológica de oficinas de formação dos manipuladores de alimentos.

Além disso, é importante ressaltar a necessidade de desenvolvimento de um instrumento avaliador de BP em UANE que leve em conta as características peculiares que estes locais apresentam e que as distanciam de outras Unidades de Alimentação e Nutrição quanto ao dimensionamento de pessoal, setorização, presença integral do profissional nutricionista, uso de equipamentos industriais etc.

\section{Colaboradores}

Confirmarmos que todos os autores fizeram contribuições substanciais para a elaboração deste trabalho. Os autores contribuíram nas seguintes etapas: concepção e desenho do estudo, aquisição de dados, análise e interpretação dos dados, elaboração do artigo, revisão crítica do conteúdo intelectual e aprovação final da versão a ser apresentada. 


\section{Referências}

1. Burlandy L, Gulgelmin SA. Políticas públicas em alimentação e nutrição no Brasil. In: Barros DC, Silva DO, Gugelmin AS, organizadores. Vigilância Alimentar e Nutricional para a Saúde Indígena. Rio de Janeiro: Fiocruz; 2007. p. 123-154.

2. Brasil. Resolução no 26 de 17 de junho de 2013. Dispõe sobre o atendimento da alimentação escolar aos alunos da educação básica no âmbito do Programa Nacional de Alimentação Escolar - PNAE. [acessado 2016 Jun 20]. Disponível em: https://www.fnde.gov. $\mathrm{br} /$ fndelegis/action/UrlPublicasAction.php?acao=abrirAtoPublico\&sgl_tipo $=$ RES\&num_ato $=00000026 \&$ seq_ato $=000 \& v 1 r \_a n o=2013 \&$ sgl_orgao $=$ FNDE $/$ MEC .

3. Food and Agriculture Organization (FAO), World Health Organization (WHO). Codex Alimentarius. Recommended International Code of Practice: General Principles of Food Hygiene. Roma: Codex Alimentarius Commission; 1999.

4. Fortuna JL. Aspectos higiênico-sanitários no preparo de carne bovina servida em refeições escolares de instituições municipais e estaduais no estado do Rio de Janeiro. Rev. Higiene Alimentar 2012; 16(95):23-32.

5. Brasil. Ministério da Educação. Resolução no 38 de 16 de julho de 2009. Dispõe sobre o atendimento da alimentação escolar e do Programa Dinheiro Direto na Escola aos alunos da educação básica. [acessado 2016 Mar 03]. Disponível em: http://portal.mec.gov.br/index.php?option $=$ com_docman\&view $=$ download $\& a-$ lias $=8166$-res038-16072009-pdf\&category_slug $=$ junho-2011-pdf\&Itemid=30192

6. Brasil. Agência Nacional de Vigilância Sanitária (Anvisa). Resolução de Diretoria Colegiada n ${ }^{\circ} 216$, de 15 de setembro de 2004. Dispõe sobre Regulamento Técnico de Boas Práticas para Serviços de Alimentação. [acessado 2016 mar 01]. Disponível em: http://www.paulinia. sp.gov.br/downloads/RDC_N_216_DE_15_DE_SETEMBRO_DE_2004.pdf

7. Stedefeldt E, Cunha DT, Silva Júnior EA, Silva SM, Oliveira ABA. Instrumento de avaliação das Boas Práticas em Unidades de Alimentação e Nutrição Escolar: da concepção à validação. Cien Saude Colet 2013; 18(4):947-953.

8. Brasil. Resolução no 38 , de 16 de julho de 2009. Dispõe sobre o atendimento da alimentação escolar e do Programa Dinheiro Direto na Escola aos alunos da educação básica. [acessado 2015 nov 14]. Disponível em: http://portal.mec.gov.br/index.php?option $=\mathrm{com}_{-}$do$\mathrm{cman} \&$ view $=$ download\&alias $=8166-$ res038-16072009 -pdf\&category_slug=junho-2011-pdf\&Itemid=30192.

9. Brasil. Agência Nacional de Vigilância Sanitária (Anvisa). Resolução de Diretoria Colegiada no 275 , de 21 de outubro de 2002. Dispõe sobre o Regulamento Técnico de Procedimentos Operacionais Padronizados aplicados aos Estabelecimentos Produtores/Industrializadores de Alimentos e a Lista de Verificação das Boas Práticas de Fabricação em Estabelecimentos Produtores/ Industrializadores de Alimentos. [acessado $2015 \mathrm{Dez}$ 10]. Disponível em: http://portal.anvisa.gov.br/wps/ wcm/connect/dcf7a900474576fa84cfd43fbc4c6735/ $\mathrm{RDC}+\mathrm{N} \% \mathrm{C} 2 \% \mathrm{BA}+275,+\mathrm{DE}+21+\mathrm{DE}+\mathrm{OUTUBRO}+-$ $\mathrm{DE}+$ 2002.pdf?MOD=AJPERES.
10. Almeida KM, Porfirio AMC, Campos MRH, Díaz MEP. Hygienic, sanitary, physical, and functional conditions of Brazilian public school food services. Rev Nutr 2014; 3(27):343-356.

11. Oliveira ABA, Cunha DT, Stedefeldt E, Capalonga R, Tondo EC, Cardoso MRI. Hygiene and good practices in school meal services: Organic matter on surfaces, microorganisms and health risks. Food Control 2014; 40:120-126.

12. Oliveira MN, Brasil ALD, Taddei JAAC. Avaliação das condições higiênico-sanitárias das cozinhas de creches públicas e filantrópicas. Cien Saude Colet 2008; 13(3):1051-1060.

13. Cardoso RCV, Góes JAW, Almeida RCC, Guimarães AG, Barreto DL, Silva SA, Figueiredo KVNA, Júnior POV, Silva EO, Huttne LB. Programa Nacional de Alimentação Escolar: há segurança na produção de alimentos em escolas de Salvador (Bahia)? Rev Nutr 2010; 5(23):801-811.

14. Rebelo TGS. Aspectos higiênico-sanitários dos serviços de alimentação dos estabelecimentos públicos de ensino fundamental de Maceió [tese]. Maceió: Universidade Federal de Alagoas; 2013.

15. Machado AD, Strapazon MA, Massing LT, Moreira DG, Possamai GA, Gabriel CM, Novais RLB. Hygiene and sanitary conditions of food services in Non-Governmental Organizations in Toledo, PR. Nutrire Rev. Soc. Bras. Aliment. Nutr 2009; 34(3):141-151.

16. Leite CL, Cardoso RCV, Góes JAW, Figueiredo KVNA, Silva EO, Bezerril MM. Formação para merendeiras: uma proposta metodológica aplicada em escolas estaduais atendidas pelo programa nacional de alimentação escolar, em Salvador, Bahia. Rev Nutr 2011; 24(2):275-285.

17. Ceccim RB. Educação permanente em saúde: descentralização e disseminação de capacidade pedagógica na saúde. Cien Saude Colet 2005; 10(4):975-986.

18. Lopes ACCL, Pinto HRF, Costa DCIO, Mascarenhas RJ, Aquino JS. Avaliação das Boas Práticas em unidades de alimentação e nutrição de escolas públicas do município de Bayeux, PB, Brasil. Cien Saude Colet 2015; 20(7):2267-2275.

19. Brasil. Agência Nacional de Vigilância Sanitária (Anvisa). Resolução de Diretoria Colegiada n ${ }^{\circ} 49$ de 31 de outubro de 2013. Dispõe sobre a regularização para o exercício de atividade de interesse sanitário do microempreendedor individual, do empreendimento familiar rural e do empreendimento econômico solidário e dá outras providências. [acessado 2016 Jun 20]. Disponível em: http://bvsms.saude.gov.br/bvs/saudelegis/ anvisa/2013/rdc0049_31_10_2013.html.

Artigo apresentado em 22/06/2016

Aprovado em 21/10/2016

Versão final apresentada em 23/10/2016 
